\title{
ERG observations of drift echoes during a unique period of the satellite mission
}

\author{
Tzu-Fang Chang ${ }^{1 *} \mathbb{D}$, Chio-Zong Cheng ${ }^{2}$, Sunny Wing-Yee Tam² ${ }^{2}$ Chih-Yu Chiang ${ }^{2}$, Yoshizumi Miyoshi ${ }^{1}$, \\ Tomoaki Hori ${ }^{1}$, Takefumi Mitani ${ }^{3}$, Takeshi Takashima ${ }^{3}$, Ayako Matsuoka ${ }^{3}$, Mariko Teramoto ${ }^{1}$ and Iku Shinohara ${ }^{3}$
}

\begin{abstract}
Substorm-associated electron injection, starting on Apr. 5, 2017, was observed by the ERG (Arase), GOES-15 and GOES-13 spacecraft. ERG successfully observed a clear and sufficient extent of manifestations of the dispersionless injection and the successive drift echoes at radial distances shorter than geosynchronous orbit (GEO) during a unique period of the satellite mission. The GOES-15 and GOES-13 measured the drift echoes of the event as well. The observations provided constraints to study the event and opportunities to make adjustments to the previous substorm injection models. Models built on an impulsive earthward-propagating electromagnetic field have been proposed to simulate substorm injections. So far such models showed good results of dispersionless features compared to spacecraft observations, but could only produce drift echoes with periods somewhat different from geosynchronous observations. To study the substorm injection event and produce drift echoes with better periods, we modify an existing model in the literature. ERG and GOES spacecraft measured tens to a few hundred keV electrons injected during the substorm, providing important seed population for ring current and radiation belts. Since the electron energies of interest are comparable to the rest mass energy, our work further provides the relativistic form of the previous model and employs a semiempirical model as background field instead of a dipole-based one in the previous study. Our work shows that the main features of the substorm injection event are successfully reproduced with the drift echoes periods showing a better fit to the observations of this event when relativistic effects are considered. Despite possible deviation of the model magnetic fields from reality, the relativistic computations still show dominant effect on the drift echoes periods. The substorm injection expanding earthward farther than GEO was observed by ERG, and the event can be better simulated by the further-developed model shown in this work.
\end{abstract}

Keywords: ERG, Arase, Substorm injection, Drift echoes, Relativistic effects

\section{Introduction}

Particle injections associated with substorms were usually studied based on observations at the geosynchronous orbit (GEO). But the Exploration of energization and Radiation in Geospace (ERG, nicknamed Arase) spacecraft also observed a substorm injection event at radial distances shorter than GEO during a special period of the mission. The magnetospheric spacecraft, ERG, was successfully launched by the Epsilon launch vehicle from the JAXA Uchinoura Space Center on

\footnotetext{
*Correspondence: jocelyn@isee.nagoya-u.ac.jp

${ }^{1}$ Institute for Space-Earth Environmental Research, Nagoya University,

Nagoya, Japan

Full list of author information is available at the end of the article
}

December 20, 2016. The apogee and perigee altitudes of ERG orbit were $\sim 6 R_{E}$ and above $\sim 400 \mathrm{~km}$, respectively, and the orbital inclination was $\sim 31^{\circ}$ (Miyoshi et al. 2018a). So ERG specifically observed the whole radiation belts region to explore the changes in the radiation belts and dynamics of geospace storms. ERG started its regular observations in March 2017 and observed near the magnetic equator for the season. In early April, ERG still observed the inner magnetosphere near the equator. A relatively isolated substorm occurred, and a clear and sufficient evolution of the substorm injection was observed by ERG 22:30 UT on Apr. 5, 2017. 
ERG captured the evident features of a nearly dispersionless injection, hereinafter called "a dispersionless injection," and successive drift echoes. The geosynchronous spacecraft, GOES-15 and GOES-13, also observed the substorm-associated drift echoes. During substorms, electrons with energies up to a few hundred $\mathrm{keV}$ were generally injected from farther tail toward the inner magnetosphere, contributing seed populations for ring current and radiation belts (Miyoshi et al. 2013). In the cross-energy coupling process in the inner magnetosphere (Miyoshi et al. 2018a), these energetic electrons played an important role as seed population which should be accelerated to the relativistic energy (Jaynes et al. 2015). ERG observed the dispersionless injection with tens to a few hundred keV electrons. Several substorm injection models were proposed to unveil the energization mechanisms of injected particles and reproduce manifestations of the first flux rise and drift echoes observed at GEO. The injected particles might subsequently serve as a source of $\mathrm{MeV}$ particles in the radiation belts. To investigate substorm injections, Birn et al. $(1997,1998)$ performed MHD simulations including relativistic effects to examine particles' trajectories in the dynamic fields and explained the initial rise feature of the injection at GEO. Li et al. (1998) and Zaharia et al. (2000) built their models on the idea that in a dipolar-based background $\boldsymbol{B}$ field, an electromagnetic pulse associated with dipolarization propagated earthward and led to dispersionless injections during substorms. Both studies produced main features of substorm injection observed at GEO. Li et al. (1998) constructed their model in spherical coordinates and assumed a plane-wave-like $\boldsymbol{E}$ field of the pulse. Their work followed electrons using a relativistic guiding center approximation with $v_{/ /}=0$. Zaharia et al. (2000) proposed a classical pulse model by assuming a radial pulse $\boldsymbol{E}$ waveform in cylindrical coordinates. Zaharia et al. (2004) improved their previous model by stretching the dipolar-based $\boldsymbol{B}$ field as the pulse passed by. Zaharia et al. (2000, 2004) performed computations for nonrelativistic particles. Our work adopts the semiempirical model (Tsyganenko 2002a, b) and chooses to build our relativistic model in cylindrical coordinates. We solve the guiding center equation of motion in a pulse with a radial waveform. So in the equatorial plane, both the background field and the pulse's waveform fit the cylindrical symmetry. According to the fact of observations, the modeling results in Zaharia et al. (2000) could be improved regarding the tens-of-minute-scale drift echoes periods. Comparing with large-scale fields, e.g., convection and background fields, substorm-associated impulsive fields were smaller-scale fields. Such pulse models (substorm injection models) were usually introduced to represent small-scale fields of substorms in some models which consider magnetospheric variations at whole MLT (e.g., Ganushkina et al. 2013).

Since satellites could not consecutively observe these particles, several models were constructed to provide changes of radiation belts or global magnetosphere at all MLT and $L$ shells. The Space Weather Modeling Framework (SWMF) (Tóth et al. 2005) was set up for physics-based space weather modeling. Glocer et al. (2011) performed simulations with the SWMF arranging the models of ionospheric dynamics, ring current, radiation belt and global magnetosphere. Ganushkina et al. (2013) utilized the Inner Magnetosphere Particle Transport and Acceleration model (IMPTAM) (Ganushkina et al. 2005) to model two storm events. Their work included the small-scale substorms fields in addition to largescale configurations. They applied the substorm injection model of $\mathrm{Li}$ et al. (1998) to introduce electromagnetic pulses to obtain changes led by stormtime substorms. The obtained distribution of electron fluxes, i.e., the output of the IMPTAM modeling, could be an input to the radiation belts models to investigate local particle acceleration up to relativistic energies.

The combination of convection- and substorm-associated fields could be the drivers of transport of plasma sheet electrons from $10 R_{\mathrm{E}}$ to GEO (Ganushkina et al. 2013). To investigate particle transport and acceleration from plasma sheet to GEO, further development of large-scale models (e.g., background field models) and substorm injection models is necessary. In this study, we improve the substorm injection model of Zaharia et al. (2000) and apply it to simulate the substorm injection event. Since a dipolar-based background field is a simplification in investigating substorms, the discrepancy between a dipolar configuration and real magnetosphere can result in adverse modeling results. Our work adopts the T01 model and includes relativistic effects. It is shown that the main features of fluxes observed by ERG, GOES-15 and GOES-13 are successfully reproduced with better drift echoes periods by our modeling including relativistic effects.

\section{Observations and data}

The orbits of the spacecraft which observed the substorm injection occurring at 22:30 UT on April 5, 2017 are shown in Fig. 1a. Figure 1b shows the IMF $B_{z}$ turns from southward to northward. IMF $B_{z}$ data used here are from OMNIWeb service of the Space Physics Data Facility at the Goddard Space Flight Center (http://omniweb.gsfc. nasa.gov/). A sudden large increase and decrease of the $\mathrm{AE}$ and $\mathrm{AL}$ indices, respectively, are measured at the substorm onset (Fig. 1c, d), and the ASY-H index shows a positive bay during the substorm (Fig. 1e). Figure $1 \mathrm{f}$ shows the model results and observed fields measured 
by the Magnetic Field Experiment (MGF) onboard ERG (Matsuoka et al. 2018). Both $\left|\boldsymbol{B}_{\mathrm{MGF}}\right|$ and $\left|\boldsymbol{B}_{\mathrm{IGRF}+\mathrm{T} 01}\right|$ (Tsyganenko 2002a, b) are smaller than $\left|\boldsymbol{B}_{\text {IGRF }}\right|$, indicating that the field lines were stretched. Figure $1 \mathrm{~g}$ shows $B_{x}$ and $B_{z}$ components observed at ERG positions. ERG observed a nearly dispersionless, namely not perfectly dispersionless, injection accompanied by a gradual increase of $B_{z}$ instead of a sharp dipolarization. The gradual dipolarization signatures and duration were similar to the second substorm injection in Gkioulidou et al. (2015). At 22:30 UT, significant enhancement of electron differential fluxes was observed by the High-energy Electron exPeriments (HEP) (Fig. 1h) of ERG, which was orbiting near the magnetic equator at radial distances shorter than GEO. HEP is equipped with two types of telescope, HEP-L $(70 \mathrm{keV} \sim 1 \mathrm{MeV})$ and HEP-H $(0.7 \sim 2 \mathrm{MeV})$ (Mitani et al. 2018). Considering $90^{\circ}$ local pitch angle electrons, Fig. $2 \mathrm{a}-\mathrm{c}$ ) shows the electron fluxes near/ at $6.6 R_{\mathrm{E}}$ as observed by ERG, GOES-15 and GOES-13, respectively. During the period of interest, ERG was near its apogee and crossing the equatorial plane. At 22:30 UT, ERG was at $Z_{S M} \sim-1.2 R_{E}$ approaching the equatorial plane, and the HEP-L first observed a dispersionless injection at the post-midnight location (Fig. 2a). Subsequently, the injection was observed at GOES-15 and GOES-13 locations as the injected electrons drifted eastward. The Magnetospheric Electron Detector (MAGED) telescopes onboard GOES-15 and GOES-13 measured the dispersion features of the drift echoes with a $\sim 45$ - $\mathrm{min}$ period for the $\sim 275 \mathrm{keV}$ energy range for example (Fig. 2b, c). After the first sharp increases in fluxes, ERG also captured manifestations of the successive echoes with periodic intervals (depending on electron energies) (Fig. 2a). Note that Fig. 2a-c shows the observed directional fluxes for $90^{\circ}$ pitch angle electrons. ERG HEP-L data are obtained from the ERG Science Center (Miyoshi et al. 2018b) (https://ergsc.isee.nagoya-u.ac.jp/index .shtml.en). The directional fluxes of the GOES-15 and GOES-13 MAGED telescopes and their pitch angles are provided in the NOAA archival data (http://satdat.ngdc. noaa.gov/sem/goes/data/new_avg/). At 00:20 UT on April 6, 2017, ERG was crossing the equatorial plane. The injected electrons were detected more than once by each spacecraft considered in this study.

\section{Model}

Since ERG successfully observed an explicit and adequate evolution of the dispersionless injection and the successive drift echoes, it provided constraints for us to explore the event and further develop the previous model. Since the electron energy range of interest is comparable to the rest mass energy, we further include relativistic effects in computations and adopt the T01 model (Tsyganenko

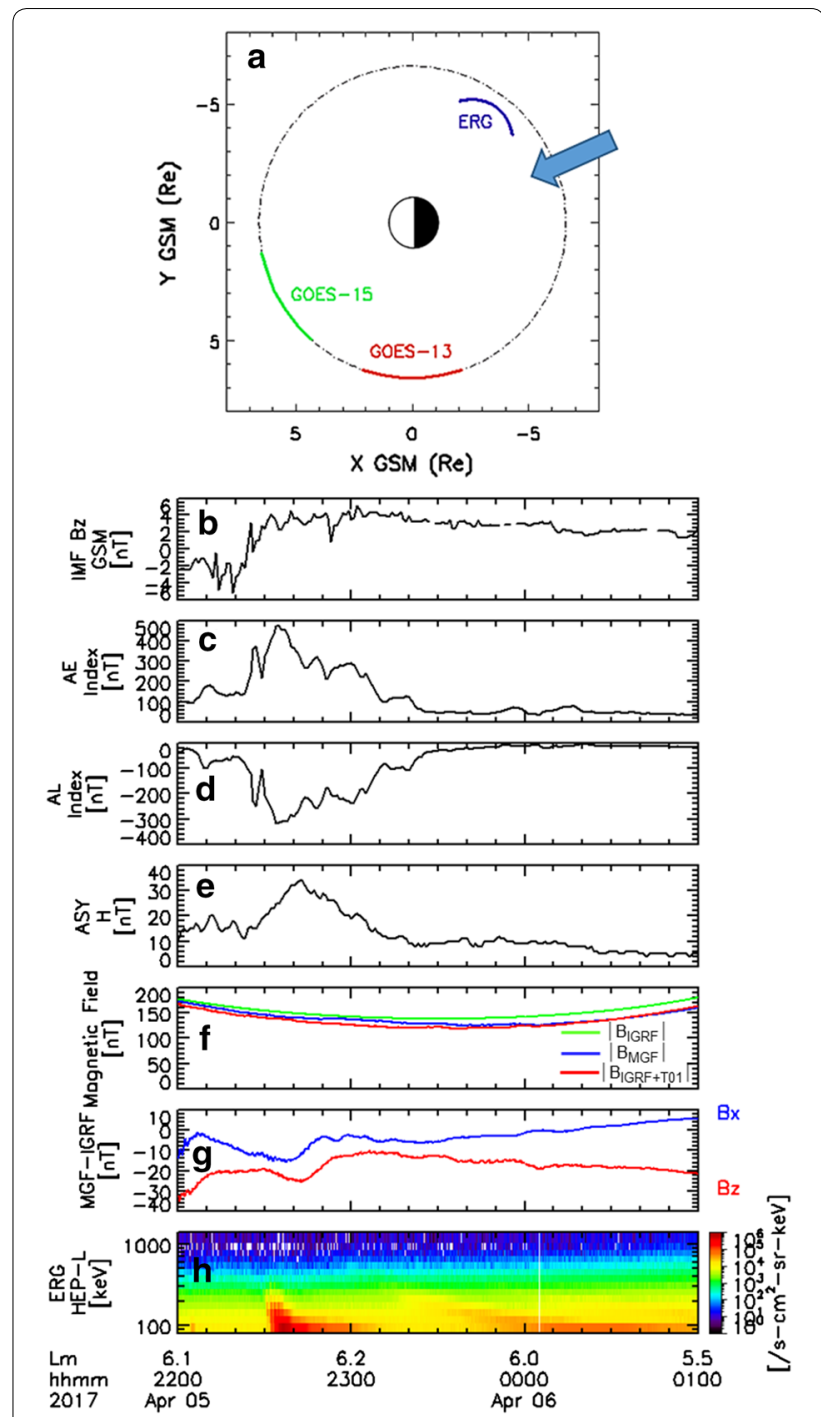

Fig. 1 a Locations of ERG, GOES-15, GOES-13 during the event initiating at $\sim 22: 30$ UT on 5 April 2017. b The variations of IMF $B_{z}$. , d, e AE, AL and ASY-H indices. $\mathbf{f}$ The magnitudes of magnetic field as indicated in the legend. $\mathbf{g} B_{x}$ and $B_{z}\left(B_{\text {IGRF }}\right.$ subtracted from $\left.B_{\text {MGF }}\right)$ in GSM coordinates $\boldsymbol{h}$ energy-time spectrograms from ERG HEP-L. Colors indicate electron differential fluxes (omni-flux data)

2002a, b) as background field instead of a dipole-based one used in Zaharia et al. (2000). Assuming an electromagnetic wave propagating earthward is formed during substorm onset, with velocity $\boldsymbol{V}_{0}$, westward $\boldsymbol{E}$ and inductive $\delta \boldsymbol{B}$ fields, the electric and magnetic fields of the pulse are coupled by Faraday's law, whose $\mathrm{z}$ component is given by

$$
\frac{\partial\left(r E_{\phi}\right)}{\partial r}=-\frac{\partial\left(r \delta B_{z}\right)}{\partial t} .
$$




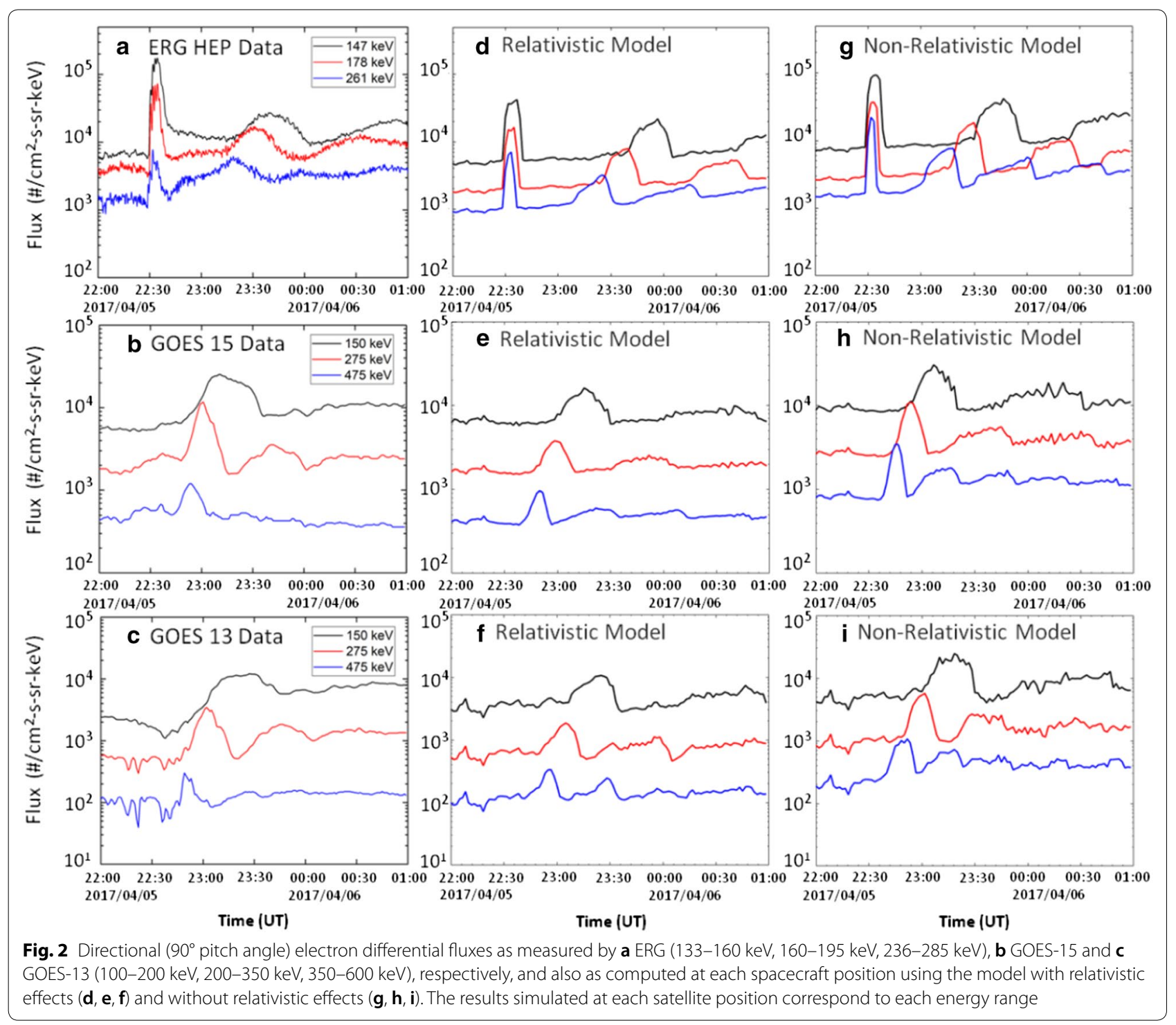

We set up the cylindrical system $(r, \phi, z)$ which has $r$ measured from the center of the Earth, $z=0$ specifying the magnetic equatorial plane and $\phi=0$ at local midnight, positive eastward. With energies in the range of interest, the electron motions in the pulse easily conserve the first adiabatic invariant. Besides, we consider $90^{\circ}$ pitch angle electrons with $v_{/ /}=0$, so curvature drift is not included in the guiding center equation of motion. The nonrelativistic form of the $\nabla B$ drift is

$$
\boldsymbol{V}_{\nabla B}=\frac{\mu}{q B^{2}} \boldsymbol{B} \times \nabla B,
$$

where $q=-e$ is the charge and $\mu \equiv m_{0} v_{\perp}^{2} /(2 B)$ is its magnetic moment. Considering relativistic effects in the substorm injection event, the $\nabla B$ drift is

$$
\boldsymbol{V}_{\phi}=\boldsymbol{V}_{\nabla B}=\frac{M_{\gamma}}{q} \frac{\boldsymbol{B} \times \nabla B}{B^{2}},
$$

where $\gamma=1 / \sqrt{1-v^{2} / c^{2}}$ is the relativistic factor, $M_{\gamma} \equiv p_{\perp}^{2} /\left(2 \gamma m_{0} B\right)$ is the magnetic moment and $m_{o}$ is the rest mass. Here $p_{\perp}=\gamma m_{0} v_{\perp}$ is the perpendicular relativistic momentum.

As moving the particle guiding centers to locations with different $\boldsymbol{B}$ intensities, the energy of electrons changes. The change in energy is due to electron guiding center motions in the $\boldsymbol{E}$ direction with $\mu$ conserved. Considering the energy variation of electrons motions in the pulse, one can derive a conservation form for the nonrelativistic condition:

$$
\frac{\mathrm{d}}{\mathrm{d} t}\left(E_{k}-\mu B\right)=0
$$


where $E_{\mathrm{k}}=m_{0} v_{\perp}^{2} / 2$ is the kinetic energy. After integration, one obtains [Equation (9) of Zaharia et al. (2000)]

$$
E_{k f}=E_{k i} \frac{B_{f}}{B_{i}},
$$

where $E_{k f} / E_{k i}$ is the electron energy at its final/initial position with the final/initial magnetic field $B_{f} / B_{i}$. Note that $B_{f}$ is the total magnetic field (interacted with the pulse fields) at the final position. Therefore, the adiabatic change in particle energy depends only on the change in $\boldsymbol{B}$ intensity. In this work, we use the background field given by T01 model. Besides, by including relativistic effects, a conservation form is obtained as:

$$
\frac{\mathrm{d}}{\mathrm{d} t}\left(\frac{W^{2}}{2 m_{0} c^{2}}-\gamma M_{\gamma} B\right)=0
$$

where $W=E_{k}+m_{0} c^{2}=\gamma m_{0} c^{2} \quad$ is the total energy. $E_{\mathrm{k}}$ is the electron energy on the equatorial plane. Integrating Eq. (6) with $M_{\gamma}=p_{\perp}^{2} /\left(2 \gamma m_{0} B\right)=\left(E_{k}^{2}+2 E_{k} m_{0} c^{2}\right) / 2 \gamma m_{0} c^{2} B$, the relation between the electron energy and the magnetic field is then given by

$$
E_{k f}=m_{0} c^{2}\left(\sqrt{1+\frac{\gamma_{\mathrm{f}}}{\gamma_{\mathrm{i}}} \frac{B_{f}}{B_{i}}\left(\frac{E_{k i}^{2}}{m_{0}^{2} c^{4}}+\frac{2 E_{k i}}{m_{0} c^{2}}\right)}-1\right) .
$$

When the kinetic energy is much less than the rest mass energy, one can reproduce the nonrelativistic approximation: $E_{k f} \approx E_{k i} B_{f} / B_{i}$.

Solving Eq. (1) in an earthward-propagating pulse with velocity $V_{0}$, one obtains the general solutions in the following form:

$$
\begin{aligned}
& r E_{\phi}=-\varepsilon_{0} \Re\left(r+V_{0} t\right) \Phi(\phi) \\
& \delta B_{z} \widehat{e_{z}}=-\frac{E_{\phi}}{V_{0}} \widehat{e_{z}},
\end{aligned}
$$

where $\varepsilon_{0}$ is a constant with the dimension of distance times electric field, $\Re\left(r+V_{0} t\right)$ is a radial wave form and $\Phi(\phi)=1$ inside a wedge (centered at an arbitrary $\phi_{0}$ ) and of finite azimuthal extent $2 \delta \phi$ and $\Phi(\phi)=0$ elsewhere. In the nonrelativistic theory, the radial and azimuthal equations of motions for guiding centers of electrons moving in the pulse are

$$
\begin{aligned}
& \frac{\mathrm{d} r}{\mathrm{~d} t}=\frac{-V_{0} \varepsilon_{0} \Re}{V_{0} r|\boldsymbol{B}|+\varepsilon_{0} \Re} \\
& \frac{\mathrm{d} \phi}{\mathrm{d} t}=\frac{\mu}{q} \frac{-3 V_{0} \frac{|\boldsymbol{B}|}{r}+\varepsilon_{0}\left(\frac{\partial \Re}{\partial r} \frac{1}{r}-\frac{\Re}{r^{2}}\right)}{V_{0} r|\boldsymbol{B}|+\varepsilon_{0} \Re} .
\end{aligned}
$$

Considering relativistic effects on the motion for electrons guiding centers in the fields of the pulse, the azimuthal equation of motion is then given by

$$
\frac{\mathrm{d} \phi}{\mathrm{d} t}=\frac{M_{\gamma}}{q} \frac{-3 V_{0} \frac{|\boldsymbol{B}|}{r}+\varepsilon_{0}\left(\frac{\partial \Re}{\partial r} \frac{1}{r}-\frac{\Re}{r^{2}}\right)}{V_{0} r|\boldsymbol{B}|+\varepsilon_{0} \Re} .
$$

Based on Eqs. (10), (11) and (12), we perform the numerical simulations to investigate the motion for electrons guiding centers moving in the pulse.

For electrons with $v_{/ /}=0$, we compute the directional flux $j$ (Roederer 1970). With Liouville's theorem which states phase space density conservation along the trajectory, Zaharia et al. $(2000,2004)$ obtained the directional flux $j$ for the nonrelativistic particles. Since our work includes relativistic effects, we consider

$$
\frac{\mathrm{d} W}{\mathrm{~d} v}=\frac{m_{0} v}{\left(1-(v / c)^{2}\right)^{3 / 2}} .
$$

Thus, the flux regarding relativistic effects becomes

$$
\mathrm{j}=\frac{c}{4 \pi}\left(\frac{\gamma_{i}}{\gamma}\right)^{3} \frac{1-\left(\frac{1}{\gamma}\right)^{2}}{\sqrt{1-\left(\frac{1}{\gamma_{i}}\right)^{2}}} f\left(W_{i}, r_{i}, t_{i}\right),
$$

where $f\left(W_{i}, r_{i}, t_{i}\right)$ is the distribution function [see Eq. (15)]. Particles with other pitch angles were assumed to be energized in the same degree in Zaharia et al. (2000, 2004) in order to make a comparison between the computed and the observed omnidirectional flux. Our work compares the $90^{\circ}$ pitch angle electron differential fluxes as measured by ERG, GOES-15, GOES-13 with those as modeled by including/excluding relativistic effects.

\section{Results and discussions}

ERG observed the entire radiation belts, so it provided critical data for developing models which gave variations of radiation belts or inner magnetosphere at all MLT and $L$ shells. Besides, ERG observed a substorm injection event, occurring at 22:30 UT on April 5, 2017, near the magnetic equator. Substorm injections were usually studied based on geosynchronous observations previously (e.g., Chang et al. 2012). ERG observed the event at radial distances shorter than GEO indicating the substorm injection expanded farther than GEO. ERG successfully captured the dispersionless feature and drift echoes of the substorm injection event, which showed an explicit and sufficient extent of manifestations and thus provided constraints for us to further develop the previous model. The tens to a few hundred keV electrons observed by ERG and GOES spacecraft were injected from farther tail 
during the substorm, providing seed populations for ring current, radiation belts and free energy for wave-particle interactions (Miyoshi et al. 2013).

The results for the substorm injection event (Fig. $2 \mathrm{~d}-\mathrm{i}$ ) are achieved with the pulse parameters $\varepsilon_{0}=4.4 \mathrm{mV} / \mathrm{m} \times 9 R_{\mathrm{E}}, \quad V_{0}=200 \mathrm{~km} / \mathrm{s}, \quad 2 \delta \phi=30^{\circ}$ and $\Delta_{1}=\Delta_{2}=\Delta_{3}=8000 \mathrm{~km}$ (Fig. 3), similar to those adopted in Zaharia et al. (2000). This approach requires less parameters compared to previous studies (e.g., Li et al. 1998). So with limited parameters, the approach is easier to clarify the dependence of physical processes on the parameters. The initial electron distribution is modeled by a kappa distribution in energy (Vasyliunas 1968):

$$
f(W, r)=f_{0} \cdot g(r)\left[1+\frac{W}{(\kappa-1.5) \varepsilon}\right]^{-\kappa-1}
$$

with average energy being $\varepsilon$, a parameter $\kappa$, a factor $f_{0}$ and the radial dependence $g(r)$. To keep with a similar condition reported by previous studies, we include the form of radial dependence $g(r)=\left(r / R_{E}-3\right)^{4} /\left(r / R_{E}\right)^{10}$ (Li et al. 1998; Zaharia et al. 2000) and it is assumed to be uniform in local time. We choose $\kappa=5$ and $=2 \mathrm{keV}$ for electrons for a moderate plasma sheet (Christon et al. 1991). Assuming a typical density of $\sim 1 \mathrm{~cm}^{-3}$ (Vasyliunas 1968), these parameters bring about $f_{0}=15 \mathrm{~km}^{-6} \mathrm{~s}^{3}$ (Vasyliunas 1968; Birn et al. 1998; Kim et al. 2000).

Figure 2 displays a comparison of electron differential fluxes for three energy ranges, as observed by ERG, GOES-15 and GOES-13 near/at GEO (left column) and also as simulated at each satellite position using the models including/excluding relativistic effects (center/right column). In the relativistic and nonrelativistic modeling, electrons are injected from the same direction (pulse centered at 1.8 MLT). It shows that both the relativistic modeling and nonrelativistic modeling reproduce the major evolution of fluxes in the event, such as dispersionless/dispersion characteristics and drift echoes,

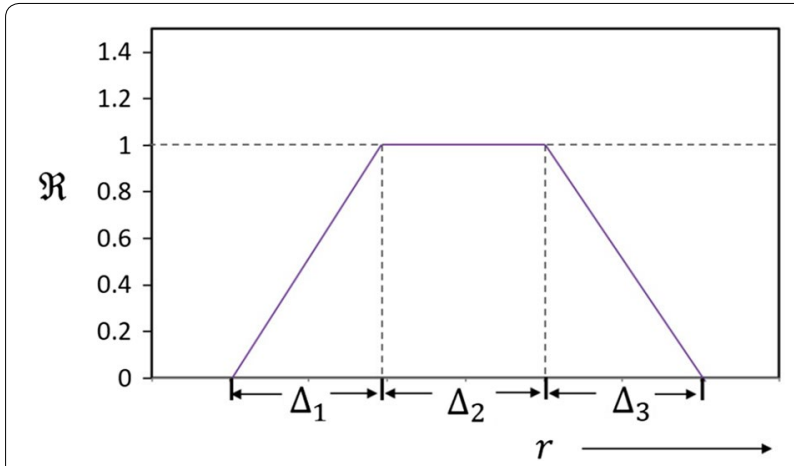

Fig. 3 Pulse form of $\Re$ along the $r$ direction even applying such a simplified pulse form. Note that the spacecraft motions are taken into account in computation. Comparing the relativistic modeling results with the nonrelativistic ones, we find the periods of drift echoes simulated with relativistic effects are different from the ones obtained without considering relativistic effects. Regarding the flux evolution for the energy $178 \mathrm{keV}$ in Fig. 2d, for example, the relativistic modeling results simulated at ERG positions provide a good fit to the first sharp flux increase as well as two subsequent drift echoes observed by ERG (Fig. 2a). However, for the same interval, the nonrelativistic modeling pictures one more drift echo (Fig. 2g) in addition to the first flux rise and two successive drift echoes. Similarities and differences in the produced flux evolution can also be found for the other energy bands shown in Fig. 2d, g). The modeling results excluding relativistic effects produce shorter drift echo periods compared to the ones including relativistic effects. The analogous effect upon drift echoes periods is also found in the results simulated at GOES spacecraft positions. The electrons kept drifting and were measured by GOES-15 and GOES-13. In regard to the observations for the $\sim 275 \mathrm{keV}$ by GOES-15, three drift echoes were observed as shown in Fig. 2b. The simulation with relativistic effects shows corresponding drift echoes periods (Fig. 2e), while the one without relativistic effects depicts more than three drift echoes (Fig. 2h), i.e., echoes with shorter periods. The differences in drift echo periods are also reflected for the other energy bands in Fig. 2e, h) as well as for the ones shown in Fig. 2f, i which are simulated at GOES-13 positions. The relativistic computations prolong the drift echo periods compared to the ones via nonrelativistic computations. The drift echoes periods simulated with relativistic effects fit better to the spacecraft observations in the injection event.

To examine the differences, we consider the relation between the azimuthal motion and the kinetic energy. Note that the injected electrons were measured more than once by each satellite while the pulse interacts with the injected electrons only once in our model. Figure 4a shows that $\nabla B$ drift velocity is reduced as including relativistic effects. We also find the ratio of the relativistic $\nabla B$ drift velocity versus the nonrelativistic one decreases with the increasing kinetic energy (Fig. 4a). Therefore, the relativistic computations produce the main features in flux evolution with prolonged drift echoes periods compared to the ones obtained without relativistic effects. Note that the azimuthal motions, which affect how long the injected electrons stay in the pulse, also depend on the magnetic moment and the azimuthal extent of the pulse which relates the pulse parameters. Basically, electrons remain in the pulse for longer time with a lower pulse velocity $V_{0}$ and a larger radial pulse length. Or in 

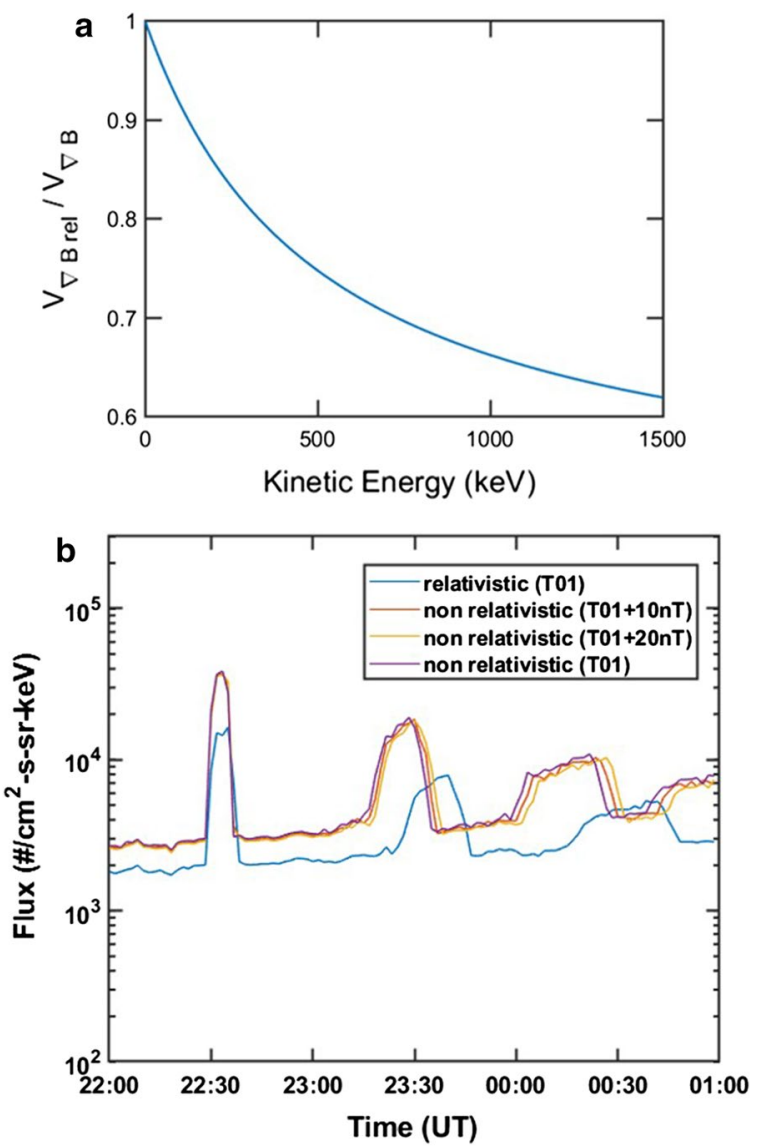

Fig. 4 a Ratio of the relativistic $\nabla B$ drift velocity to the nonrelativistic one versus the kinetic energy. $\mathbf{b}$ Influences of relativistic effects and the magnetic fields (as indicated in the legend) exerted on drift echoes periods strong electric field condition, the larger $\boldsymbol{E} \times \boldsymbol{B}$ drift can get close to the $V_{0}$, enabling the electrons to remain in the pulse longer. Besides, magnetic field also influences the azimuthal motions. Considering the possible differences of model magnetic fields and the observed fields as shown in Fig. 1f, relativistic effects still play a dominant role in varying drift echoes periods (Fig. 4b).

The other main difference between the relativistic computations and the nonrelativistic ones is the preinjection flux level. The apparent differences are shown in Fig. 4b too. In Fig. $2 \mathrm{~d}-\mathrm{i}$, it shows that the pre-injection fluxes computed with relativistic effects get lowered compared to those obtained by nonrelativistic theory. We investigate the relations between electron flux variation and kinetic energy before and after acceleration. Before the acceleration, i.e., $W_{f}=W_{i}, \quad r_{f}=r_{i}$, the ratio of relativistic electron flux versus nonrelativistic one decreases with the increasing kinetic energy (Fig. 5a). As for after the electrons are accelerated, i.e., $W_{f}>W_{i}, r_{f}>r_{i}$, we find the ratio of relativistic electron flux versus nonrelativistic electron flux decreases with the increasing ratio of $W_{f}$ to $W_{i}$ (Fig. 4b). Besides, for larger $W_{i}$, the ratio of the relativistic electron flux to the nonrelativistic one decreases more with the increasing ratio of $W_{f}$ to $W_{i}$ (Fig. 5b). With the dependence of electron flux upon kinetic energy and initial/ final energy of the electrons, we suggest the relativistic computations in our model produce lower pre-injection flux levels compared to the ones by the nonrelativistic computations, as shown in Fig. 2-i. Note that we adopt T01 model and set up a background $\boldsymbol{E}$ field $(0.2 \mathrm{mV} / \mathrm{m})$ (Christon et al. 1991). So before the injection, motions of electrons in the cells considered could
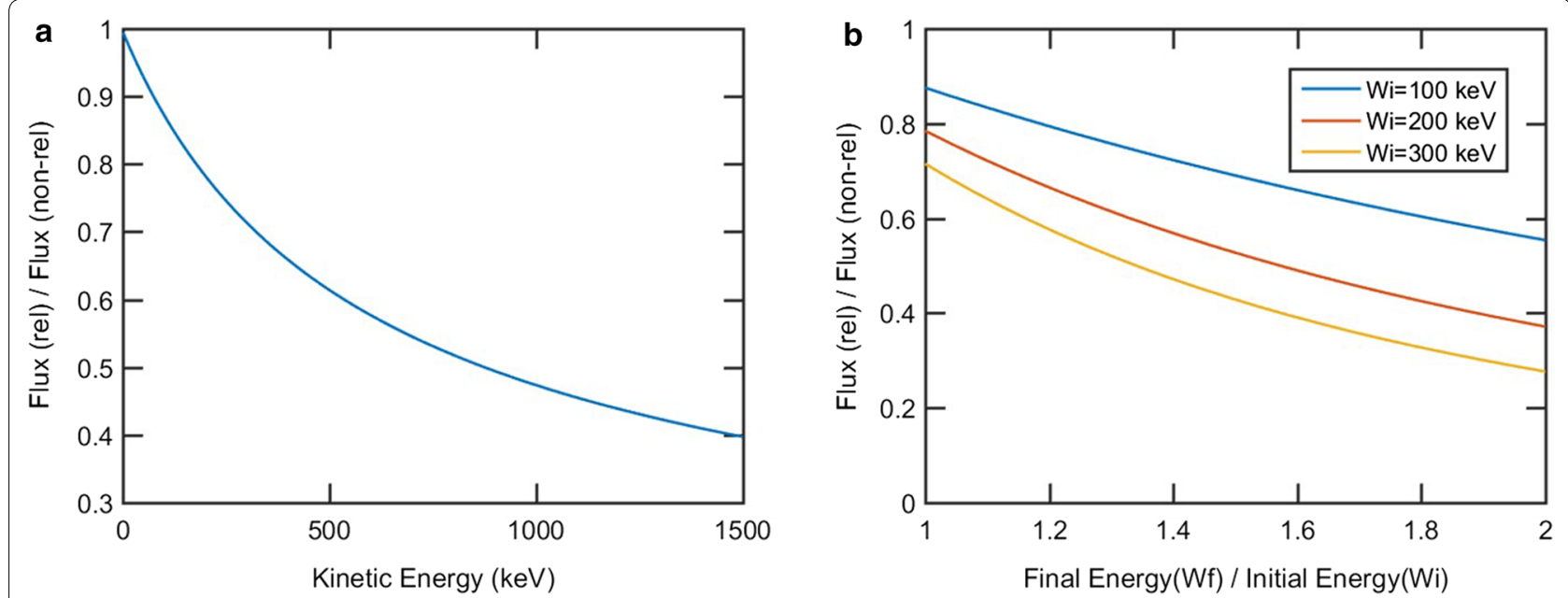

Fig. 5 a Ratio of the relativistic electron flux to the nonrelativistic one versus the kinetic energy (before acceleration). $\mathbf{b}$ The ratio of the relativistic electron flux to the nonrelativistic one versus the ratio of electron final energy to electron initial energy for three energy ranges 
be affected by the background $\boldsymbol{E}$ and time-dependent $\boldsymbol{B}$ fields. Besides, the small-scale fluctuations in Fig. $2 \mathrm{~d}-\mathrm{i}$ could also be caused by the finite number of cells considered and the time step used in plotting the data.

The impulsive fields of the substorms play a role in transporting and accelerating electrons from the plasma sheet to the inner magnetosphere. So fields of substorms must be taken into consideration regarding electron populations modeling (Ganushkina et al. 2013). During a substorm, electrons with energies of a few hundred $\mathrm{keV}$ can be swept by an earthward-propagating pulse in a time-dependent background field to higher magnetic field strength locations during which the hundreds of $\mathrm{keV}$ electrons are energized via the betatron acceleration process which is based on the magnetic moment conservation.

This work investigates how a set of parameters which describes a moderate active plasma sheet affects the injected electrons during a substorm. On the other hand, we consider $90^{\circ}$ pitch angle electrons in our model. The work in Birn et al. $(1997,2004)$ showed that $90^{\circ}$ pitch angle particles are energized the most. Nevertheless, it is satisfactory to consider further working on particles with different pitch angles in future work to reproduce the features of substorm injections in a more complete aspect. Since the injected tens to a few hundred keV electrons as observed by ERG and GOES spacecraft may become a source of energetic particles in radiation belts. The improved substorm injection model can be launched to stand for spatially localized electromagnetic pulses of substorms in models which characterize magnetospheric changes at all MLT. The simulated distributions of electron fluxes can serve as input to radiation belts models. Therefore, such models which characterize magnetospheric changes at all MLT can include the smaller-scale fields of substorms and thus provide more refined results closer to realities or to even further develop the feasible capabilities related to space weather forecast.

\section{Authors' contributions}

TFC analyzed the data and performed the modeling. CZC and SWYT discussed the modeling details. CYC, YM and TH discussed the data and modeling. TM and TT generated HEP data. AM and MT generated MGF data. IS managed ERG project. All authors read and approved the final manuscript.

\section{Author details}

${ }^{1}$ Institute for Space-Earth Environmental Research, Nagoya University, Nagoya, Japan. ${ }^{2}$ Institute of Space and Plasma Sciences, National Cheng Kung University, Tainan, Taiwan. ${ }^{3}$ Institute of Space and Astronautical Science, Japan Aerospace Exploration Agency, Sagamihara, Japan.

\section{Acknowledgements}

Not applicable.

\section{Competing interests}

The authors declare that they have no conflict of interest.

\section{Availability of data and materials}

The original data used in this study are publicly available as follows. The directional differential fluxes of the MAGED telescopes and their pitch angles are provided in the NOAA archival data (http://satdat.ngdc.noaa.gov/sem/ goes/data/new_avg/). The IMF $B_{z}$ component, AE, AL and ASY-H indices are provided by the OMNIWeb service of the Space Physics Data Facility at the Goddard Space Flight Center (http://omniweb.gsfc.nasa.gov/). Science data of ERG (Arase) satellite are obtained from ERG Science Center operated by ISAS/JAXA and ISEE/Nagoya University (https://ergsc.isee.nagoya-u.ac.jp/index .shtml.en). The Arase satellite data will be publicly available via ERG Science Center on a project-agreed schedule. This study uses HEP CDF Level 2 v01_02 and MGF CDF Level 2 v01_01.

\section{Consent for publication}

Not applicable.

\section{Ethics approval and consent to participate}

Not applicable.

\section{Funding}

YM. is supported by JSPS Grant (15H05815, 15H05747, 16H06826). This study is supported by JSPS Bilateral Open Partnership Joint Research Projects. The work by S.W.Y.T. is supported by MOST Grant MOST107-2111-M006-003 to National Cheng Kung University.

\section{Publisher's Note}

Springer Nature remains neutral with regard to jurisdictional claims in published maps and institutional affiliations.

Received: 31 October 2018 Accepted: 10 February 2019

Published online: 18 February 2019

\section{References}

Birn J et al (1997) Substorm ion injections: geosynchronous observations and test particle orbits in three-dimensional dynamic MHD fields. J Geophys Res 102:2325. https://doi.org/10.1029/96JA03032

Birn J et al (1998) Substorm electron injections: geosynchronous observations and test particle simulations. J Geophys Res 103(A5):9235. https://doi. org/10.1029/97JA02635

Birn J et al (2004) Electron acceleration in the dynamic magnetotail: test particle orbits in three dimensional magnetohydrodynamic simulation fields. Phys Plasmas 11(5):1825. https://doi.org/10.1063/1.1704641

Chang TF et al (2012) Behavior of substorm auroral arcs and Pi2 waves: implication for the kinetic ballooning instability. Ann Geophys 30:911-926. https://doi.org/10.5194/angeo-30-911-2012

Christon SP et al (1991) Spectral characteristics of plasma sheet ion and electron populations during disturbed geomagnetic conditions. J Geophys Res 96:1. https://doi.org/10.1029/90JA01633

Ganushkina NYu et al (2005) Role of substorm-associated impulsive electric fields in the ring current development during storms. Ann Geophys 23:579-591. https://doi.org/10.5194/angeo-23-579-2005

Ganushkina NY et al (2013) Transport of the plasma sheet electrons to the geostationary distances. J Geophys Res Space Phys 118:82-98. https:// doi.org/10.1029/2012JA017923

Gkioulidou M et al (2015) Spatial structure and temporal evolution of energetic particle injections in the inner magnetosphere during the 14 July 2013 substorm event. J Geophys Res Space Phys 120:1924-1938. https:// doi.org/10.1002/2014JA020872

Glocer A et al (2011) Rapid rebuilding of the outer radiation belt. J Geophys Re. 116:A09213. https://doi.org/10.1029/2011JA016516

Jaynes AN et al (2015) Source and seed populations for relativistic electrons: their roles in radiation belt changes. I Geophys Res Space Phys 120:7240-7254. https://doi.org/10.1002/2015JA021234

Kim HJ et al (2000) Can substorms produce relativistic outer-belt electrons? J Geophys Res 105:7721-7736. https://doi.org/10.1029/1999JA900465

$\mathrm{Li} X$ et al (1998) Simulation of dispersionless injections and drift echoes of energetic electrons associated with substorms. Geophys Res Lett 25:3763. https://doi.org/10.1029/1998GL900001 
Matsuoka A et al (2018) The ARASE (ERG) magnetic field investigation. Earth Planets Space 70(1):1-16. https://doi.org/10.1186/s40623-018-0800-1

Mitani T et al (2018) High-energy electron experiments (HEP) aboard the ERG (Arase) satellite. Earth Planets Space 70:77. https://doi.org/10.1186/s4062 3-018-0853-1

Miyoshi Y et al (2013) High-speed solar wind with southward interplanetary magnetic field causes relativistic electron flux enhancement of the outer radiation belt via enhanced condition of whistler waves. Geophys Res Lett 40:4520-4525. https://doi.org/10.1002/grl.50916

Miyoshi Y et al (2018a) Geospace exploration project ERG. Earth Planets Space 70:101. https://doi.org/10.1186/s40623-018-0862-0

Miyoshi Y et al (2018b) The ERG science center. Earth Planets Space 70:96. https://doi.org/10.1186/s40623-018-0867-8

Roederer J (1970) Dynamics of geomagnetically trapped radiation, vol 2. Series: physics and chemistry in space. Springer, Berlin. ISBN 978-3-642-49302-7

Tóth G et al (2005) Space weather modeling framework: a new tool for the space science community. J Geophys Res 110:A12226. https://doi. org/10.1029/2005JA011126
Tsyganenko NA (2002a) A model of the near magnetosphere with a dawndusk asymmetry: 1. Mathematical structure. J Geophys Res 107(A8):1179. https://doi.org/10.1029/2001JA000219

Tsyganenko NA (2002b) A model of the near magnetosphere with a dawndusk asymmetry: 2. Parameterization and fitting to observations. J Geophys Res 107(A8):1176. https://doi.org/10.1029/2001 JA000220

Vasyliunas V (1968) A survey of low-energy electrons in the evening sector of the magnetosphere with OGO I and OGO 3. J Geophys Res 73:2839. https ://doi.org/10.1029/JA073i009p02839

Zaharia S et al (2000) Particle transport and energization associated with substorms. J Geophys Res 105:18741. https://doi.org/10.1029/1999JA000407

Zaharia S et al (2004) Substorm injection modeling with nondipolar, timedependent background field. J Geophys Res 109:A10211. https://doi. org/10.1029/2004JA010464

\section{Submit your manuscript to a SpringerOpen ${ }^{\odot}$ journal and benefit from:}

- Convenient online submission

- Rigorous peer review

- Open access: articles freely available online

- High visibility within the field

- Retaining the copyright to your article

Submit your next manuscript at $\boldsymbol{\nabla}$ springeropen.com 